\title{
Editorial
}

\section{Organic Inorganic Hybrid Perovskite Solar Cells}

\author{
Ryan T. Wang and $\mathrm{Gu} \mathrm{Xu}$ *
}

Department of Materials Science and Engineering, McMaster University, 1280 Main St W,

Hamilton, ON L8S 4L8, Canada; wangt41@mcmaster.ca

* Correspondence: xugu@mcmaster.ca

Citation: Wang, R.T.; Xu, G. Organic Inorganic Hybrid Perovskite Solar Cells. Crystals 2021, 11, 1171. https:// doi.org/10.3390/cryst11101171

Received: 14 September 2021 Accepted: 17 September 2021 Published: 27 September 2021

Publisher's Note: MDPI stays neutral with regard to jurisdictional claims in published maps and institutional affiliations.

Copyright: (c) 2021 by the authors. Licensee MDPI, Basel, Switzerland. This article is an open access article distributed under the terms and conditions of the Creative Commons Attribution (CC BY) license (https:// creativecommons.org/licenses/by/ $4.0 /)$.
Much progress has been achieved in the commercialization of solar devices, especially perovskite solar cells, which have shown excellent transport properties and low fabrication costs [1]. The organic perovskite solar cells based on $\mathrm{MAPbI}_{3}$ and $\mathrm{FAPbI}_{3}$ showed a surprising power conversion efficiency (PCE) of more than $25 \%$, as opposed to their initial PCE of 3.8\% a decade ago, inspiring a new era for green energy development [2].

Despite the great PCE, the stability of such devices remains a major challenge. The organic perovskite would decompose in the air within several hours due to moisture attack and/or phase transition. Even with the encapsulation, the lifetime could only be extended to several thousand hours, far from enough for the commercialization. As a result, a great deal of effort has since been devoted to trying to enhance the perovskite stability [3], such as strain engineering method, interface engineering, or doping in the $\mathrm{A} / \mathrm{B} / \mathrm{X}$ sites in the $\mathrm{ABX}_{3}$ structure of perovskite [1-3]. Unfortunately, these quick trials did not extend the perovskite lifetime to any appreciable level, due not least to the debatable degradation mechanism, in particular, the influence of water molecules on the perovskite structure, in addition to the possible structural variations vis-a-vis the kinetics and thermodynamics. Therefore, such efforts were destined to be less than successful. This is what the Special Issue is all about, to fill the critical gap and propose fresh strategies accordingly, and even to extend the development to other systems and devices, such as the one involving glucose sensing [4].

Several contributions have worked from the root of the instability problem, providing inspiring fresh alternatives as potential solutions for this question. For example, Seunghyun Rhee et al. [1] provided the fundamental properties of perovskite materials and categorized the usages in various optoelectronic applications. In particular, they introduced the fundamental structure and characteristics of a halide-based perovskite layer and next highlighted the key factors for achieving high performance in each application: solar cells, light-emitting diodes, lasers, and photodetectors, which is followed by a description of the remaining challenges. Their review confirmed the high potential of perovskite as a universal material solution for a wide range of optoelectronic applications and enhanced the understanding of key properties in each application for their practical commercialization. In addition, they reviewed the prerequisite factors for those applications, which assisted the understanding of the recent progress of perovskite-based optoelectronic devices and the challenges that need to be solved for the commercialization.

Moreover, Alex F. Xu et al. [2] proposed a brand-new organic perovskite structure of suitable bandgap and stability. They discovered a stable hybrid perovskite, pyrrolidinium lead iodide $\left(\mathrm{PyPbI}_{3}\right)$, via a simple drop casting method. The formed $\mathrm{PyPbI}_{3}$ exhibited a hexagonal structure, presenting not only excellent phase stability, but also high transport properties, which indicated that $\mathrm{PyPbI}_{3}$ is an environmentally stable OIHP material of great potential to be employed in perovskite PV applications. Thus, the proposed five-membered ring-based perovskite $\mathrm{PyPbI}_{3}$ provided essential steps towards resolving the long-term instability problems of PSCs. Based on this proposal, $\mathrm{Xu}$ et al. offered multi-dimensional perovskite as a potential solution by incorporating one-dimensional (1D) perovskitoid $\mathrm{PyPbI}_{3}$ [3]. The stability measurements indicated that $1 \mathrm{D}$ perovskitoid is much more stable 
than the commonly employed FA-based perovskite, whose device lifetime can be enhanced to more than one month after adding $\mathrm{PyPbI}_{3}$ as an absorbing layer, achieving huge progress towards the commercialization of PSCs. Based on the results of XRD, X-ray photoelectron spectroscopy (XPS), and FTIR, it was discovered that the hexagonal structure of $\mathrm{PyPbI}_{3}$, as well as the interatomic force between $\mathrm{Py}$ cation and $\mathrm{PbI}_{2}$ lattice, played an important role in the structural stability. Due to the strong interatomic forces between $\mathrm{Py}^{+}$cations and inorganic cages, the low dimensional perovskite exhibited excellent environmental stability compared to the 3D perovskite, providing new inspiration to the advancement of perovskite solar cells design.

The present Special Issue on "Organic Inorganic Hybrid Perovskite Solar Cells" is of huge significance for the wide PSCs research community, as it provides a fresh structural maneuvering engineering method under the current materials framework by structural physics negotiation. A novel structure of enhanced lifespan and comparable bandgap of the favorite $\alpha-\mathrm{FAPbI}_{3}$ has thus been synthesized, which provides not only thermodynamic but also kinetical warrant, extending the perovskite lifetime by more than 10-fold. Hopefully, these discoveries will be of help to the advance of PSC design and materials research in general.

Author Contributions: Writing—original draft preparation, R.T.W.; writing—review and editing, G.X. All authors have read and agreed to the published version of the manuscript.

Funding: This research received no external funding.

Conflicts of Interest: The authors declare no conflict of interest.

\section{References}

1. Rhee, K.S.; An, K.; Kang, K.T. Recent advances and challenges in halide perovskite crystals in optoelectronic devices from solar cells to other applications. Crystals 2021, 11, 39. [CrossRef]

2. Xu, A.F.; Wang, R.T.; Yang, L.W.; Liu, E.E.; Xu, G. An environmentally stable organic-Inorganic hybrid perovskite containing py cation with low trap-state density. Crystals 2020, 10, 272. [CrossRef]

3. Xu, F.; Li, Y.; Liu, N.; Han, Y.; Zou, M.; Song, T. 1D perovskitoid as absorbing material for stable solar cells. Crystals 2021, 11, 241. [CrossRef]

4. Yang, L.W.; Liu, E.E.; Xu, A.F.; Chen, J.Y.; Wang, R.T.; Xu, G. Improving linear range limitation of non-enzymatic glucose sensor by $\mathrm{OH}-$ concentration. Crystals 2020, 10, 186. [CrossRef] 